\title{
Two Functionally Distinct Networks of Gap Junction- Coupled Inhibitory Neurons in the Thalamic Reticular Nucleus
}

\author{
Seung-Chan Lee, Saundra L. Patrick, Kristen A. Richardson, and Barry W. Connors \\ Department of Neuroscience, Division of Biology and Medicine, Brown University, Providence, Rhode Island 02912
}

\begin{abstract}
Gap junctions (GJs) electrically couple GABAergic neurons of the forebrain. The spatial organization of neuron clusters coupled by GJs is an important determinant of network function, yet it is poorly described for nearly all mammalian brain regions. Here we used a novel dye-coupling technique to show that GABAergic neurons in the thalamic reticular nucleus (TRN) of mice and rats form two types of GJ-coupled clusters with distinctive patterns and axonal projections. Most clusters are elongated narrowly along functional modules within the plane of the TRN, with axons that selectively inhibit local groups of relay neurons. However, some coupled clusters have neurons arrayed across the thickness of the TRN and target their axons to both first- and higher-order relay nuclei. Dye coupling was reduced, but not abolished, among cells of connexin 36 knock-out mice. Our results suggest that GJs form two distinct types of inhibitory networks that correlate activity either within or across functional modules of the thalamus.
\end{abstract}

Key words: dye-coupling; electrical coupling; electrical synapses; gap junctions; thalamic reticular nucleus

\section{Introduction}

Gap junction (GJ)-mediated electrical synapses frequently interconnect inhibitory interneurons in circuits of the mammalian forebrain (Galarreta and Hestrin, 2001a; Connors and Long, 2004). GJs allow intercellular current flow and are involved in diverse forms of electrical signaling, such as synchronization of subthreshold and spike-mediated activity, detection of input synchrony, and modulation of firing rate (Beierlein et al., 2000; Galarreta and Hestrin, 2001b; Deans et al., 2002; Long et al., 2004; Hjorth et al., 2009; Vervaeke et al., 2010). Electrical coupling of GABAergic neurons by GJs generates coordinated inhibitory synaptic drive that can entrain and synchronize the spiking of principal cells in diverse ways (Deans et al., 2001; Traub et al., 2001; Buhl et al., 2003; Dugué et al., 2009).

The power of GJs to coordinate neuronal activity should be critically dependent on the spatial organization of coupled neu-

Received Feb. 6, 2014; revised Aug. 18, 2014; accepted Aug. 24, 2014.

Author contributions: S.-C.L. and B.W.C. designed research; S.-C.L. and S.L.P. performed research; S.-C.L. and K.A.R. analyzed data; S.-C.L. and B.W.C. wrote the paper.

This work was supported by National Institutes of Health Grants NS025983 and NS050434 and National Science Foundation Grant EFRI-0937848. We thank Scott Cruikshank for help with recording and lentivirus injections, Yifan Anna Zheng for technical assistance early in this work, Minseok Choi, Jaeyoung Sung, and Eren Mehmet Kiral for advice in data analysis, and members of Connors laboratory for their helpful comments.

The authors declare no competing financial interests.

Correspondence should be addressed to either of the following: Seung-Chan Lee, Center for Molecular and Behavioral Neuroscience, Rutgers, The State University of New Jersey, Newark, NJ 07102, E-mail: seungchannlee@gmail.com; or Barry W. Connors, Department of Neuroscience, Box G-LN, Brown University, Providence, RI 02912, E-mail: bc@brown.edu.

S.-C. Lee's present address: Center for Molecular and Behavioral Neuroscience, Rutgers, The State University of New Jersey, Newark, NJ 07102.

K. A. Richardson's present address: Galenea, Wakefield, MA 01880.

DOI:10.1523/JNEUROSCI.0562-14.2014

Copyright $\odot 2014$ the authors $\quad 0270-6474 / 14 / 3413170-13 \$ 15.00 / 0$ ronal clusters, including their size and geometric patterns relative to the patterns of their inputs and outputs. Independently coupled clusters of inhibitory neurons with different GJ connectivity could introduce distinct patterns of correlated inhibition on their downstream targets that would allow rich control over spike rate and coherence (Tiesinga et al., 2008). Despite its importance, the spatial organization of GJ-coupled networks is poorly understood in almost all parts of the brain.

In principle, a powerful technique to reveal the spatial organization of GJ-coupled networks is dye coupling, which depends on the diffusion of small dye molecules through GJ channels. However, the use of this technique in most brain regions has been limited (Stewart, 1978; Connors et al., 1983; Hampson et al., 1992; Peinado et al., 1993) as a result of shortcomings of the method. These include false-positive signals (i.e., spurious staining of uncoupled cells) caused by dye leakage from microelectrodes during injection (Connors et al., 1984; Gutnick et al., 1985; Peinado et al., 1993), as well as false negatives attributable to insufficient dye injection and sensitivity. Here we describe a new dye injection method that overcomes these technical problems and allows high-concentration intracellular dye injection without leakage extracellularly. We have used this to characterize the spatial patterns of electrically coupled neurons in the thalamic reticular nucleus (TRN).

The TRN is a thin shell of GJ-connected GABAergic neurons on the lateral aspect of the dorsal thalamus (Landisman et al., 2002; Pinault, 2004). As the major source of feedback and feedforward inhibition to thalamic relay nuclei, the TRN plays a critical role in the spatiotemporal processing of activity passing bidirectionally between thalamus and neocortex. Evidence suggests that TRN neurons in major sensory sectors are composed of thin slab-like domains that are further organized into sublaminae 
that preserve the topography of associated relay nuclei (Crabtree, 1992; Bourassa et al., 1995; Pinault et al., 1995; Kimura et al., 2005).

Our results reveal that TRN neurons form GJ-coupled clusters with two distinct spatial patterns associated with different axonal projection patterns and domains of the TRN. This suggests that GJ-coupled neuron clusters participate in two functionally distinct networks of the TRN that correlate activity within and across thalamic modules.

\section{Materials and Methods}

Slice preparation and whole-cell recording. Thalamocortical (TC) slices (Agmon and Connors, 1991) or horizontal (Hz) slices, $350 \mu \mathrm{m}$ thick, were prepared from Sprague Dawley rats [postnatal day 9 (P9) to P14, males] or wild-type (WT) or connexin36 knock-out (Cx36 KO) littermate mice (P10-P14, of either sex) (Deans et al., 2001). Cx36 KO mice were crossed into C57BL/6 background (more than N7). F1 hybrid females from crossing of Cx36 heterozygotes in C57BL/6 background and Fvb mice were obtained. Pups from crossing Cx36 heterozygotes in C57BL/ 6 background and F1 background were used for experiments. In some experiments, WT pups came from crossing C57BL/6 mice and F1 background WT mice. After deep anesthesia was achieved, animals were decapitated, and brains were removed and chilled in ice-cold artificial CSF (ACSF) and then sectioned into 350- $\mu$ m-thick slices containing the ventrobasal thalamus (VB). After sectioning, slices were incubated in $\mathrm{ACSF}$ at $32^{\circ} \mathrm{C}$ for $30 \mathrm{~min}$ and then kept at room temperature until they were transferred to a submersion-type recording chamber perfused with $\mathrm{ACSF}$ at $32^{\circ} \mathrm{C}$. Cells were visualized using infrared differential interference contrast (DIC) optics. The ACSF contained the following (in mM): $126 \mathrm{NaCl}, 3 \mathrm{KCl}, 1.25 \mathrm{NaH}_{2} \mathrm{PO}_{4}, 26 \mathrm{NaHCO}_{3}, 2 \mathrm{CaCl}_{2}, 10$ dextrose, and $2 \mathrm{MgCl}_{2}$ (saturated with $95 \% \mathrm{O}_{2} / 5 \% \mathrm{CO}_{2}$ ). For paired whole-cell recordings, micropipettes were filled with a standard internal solution that was composed of the following (in mM): $130 \mathrm{~K}$-gluconate, $0.2 \mathrm{EGTA}, 4 \mathrm{KCl}, 2$ $\mathrm{NaCl}, 10$ HEPES, 4 ATP-Mg, 0.3 GTP-Tris, and 14 phosphocreatineTris, pH 7.20-7.30 (285-295 mOsm).

Recordings were performed in current clamp using Axoprobe or Axoclamp 2B amplifiers (Molecular Devices). Data were collected and analyzed using National Instruments hardware and software. To estimate the strength of electrical coupling, 5-20 hyperpolarizing current pulses (approximately -200 to $-400 \mathrm{pA}, 600 \mathrm{~ms}$ ) were first injected into one cell, and the mean steady-state voltage deflection in that cell $\left(\Delta V_{1}\right)$ and in its coupled neighbor $\left(\Delta V_{2}\right)$ were measured. Neurons of $<15 \mu \mathrm{m}$ separation (center-to-center) in the somatosensory sector of the TRN were chosen for paired recordings. A coupling coefficient was then calculated as $\Delta V_{2} / \Delta V_{1}$. Pairs were considered to be electrically coupled if their coupling coefficient was $\geq 0.01$. Input resistances were measured from voltage responses to small negative current injections soon after achieving a whole-cell configuration. For comparisons of electrical coupling results between $\mathrm{Cx} 36 \mathrm{KO}$ and $\mathrm{WT}$ animals, the experimenters were blind to the genotypes of the mice during the recordings.

Dye injection into neurons. Neurobiotin (NB) was injected into neurons during whole-cell recording in current-clamp mode. To avoid extracellular dye leakage during the approach of pipettes to target neurons, micropipettes were front-filled with a small volume $(\sim 0.3 \mu \mathrm{l})$ of standard internal solution containing no NB and then backfilled with an internal solution containing $\mathrm{NB}$ and the fluorescent dye Lucifer yellow $\mathrm{CH}$ (LY, dipotassium salt). The NB-containing internal solution was composed of the following (in $\mathrm{mM}$ ): 56.5 K-gluconate, 77.45 Neurobiotin-Cl (2.5\%, w/v), 2 NaCl, 10 HEPES, 4 ATP-Mg, 0.3 GTPTris, and 14 phosphocreatine-Tris, pH 7.20-7.30 (285-295 mOsm). LY $(0.1 \%, \mathrm{w} / \mathrm{v})$ was dissolved in NB-containing internal solution freshly before recording.

Although micropipette tips were approaching target neurons in the brain slice, slight positive pressure was applied to the micropipette to keep the tip surface clean. Gigaohm seal formation between the micropipette tip and neuronal membrane was made before the NB-containing backfilling solution reached the tip of micropipettes (usually within 2-3 min after positive pressure application). The fluorescence of LY was used to estimate the concentration of dye-containing backfilling solution at the tip area of micropipette, with the assumption that NB and LY behave similarly during pressure-driven flow and diffuse at similar rates during the waiting period.

Absence of dye at the tip of pipette was confirmed immediately before and after gigaseal formations by checking the level of fluorescence signal in the pipette tips. Only neurons with no detectable or below minimal level of LY signal in pipette tip area were used. In most of the cases analyzed, no visible LY fluorescence was detected. In two cases, a very low level of LY $(<0.01 \% \mathrm{NB}$ equivalent) was observed in the tip area after cell attachment, and these slices were processed because this concentration of $\mathrm{NB}$ is low enough to cause no detectable false-positive staining. If a more significant level of LY fluorescence (more than $\sim 0.01 \% \mathrm{NB}$ ) was observed in the tip after gigaseal formation, those brain slices were discarded.

In control experiments, NB injection with normal internal solution containing $0.01-0.02 \% \mathrm{NB}$, but without using the front-filling technique, gave only a faintly stained single injected neuron without any other cells labeled nearby. LY fluorescence intensity was calibrated by measuring LY signals from TRN neurons injected with LY-containing internal solution at known dilute concentrations $(0.005-0.02 \%)$ at a fixed light intensity and gain of the image processor for each light lamp. A best-fit line was obtained from linear regression of the data points. Relative LY concentration of injected neurons was estimated from this calibration curve. NB concentration ("NB equivalent") was estimated assuming the relative concentration changes of NB and LY were proportional. We assumed that diffusion rates of $\mathrm{NB}$ and LY are closely similar. We estimated the diffusion coefficient $(D)$ of $\mathrm{NB}$ and $\mathrm{LY}\left(D_{\mathrm{NB}}=449\right.$, $D_{\mathrm{LY}}=428 \mu \mathrm{m}^{2} / \mathrm{s}$ ) by considering their molecular weights and the known $D$ values of five different tracers that are similar in size to NB and LY (Kamholz et al., 2001; Petrásek and Schwille, 2008). The $D_{\mathrm{NB}}$ and $D_{\mathrm{LY}}$ estimates are approximately consistent with predictions from the StokeEinstein equation. We then used Fick's diffusion equations to simulate the time course of NB and LY concentrations in a front-filled pipette tip during pipette approach stage $(<5$ min from loading the backfilling solution to cell patching). The results showed that the tip concentrations of NB and LY track each other quite closely, even when larger $D_{\mathrm{NB}}$ and $D_{\text {LY }}$ differences are assumed. They also implied that, under our conditions, false positives caused by significant NB entering the pipette tip were easily avoided. Any NB and LY concentration differences that might occur between cell attachment of the pipette and dye injection in the whole-cell configuration would apply consistently to all our injections.

In most cases, cell-attached recording mode (in gigaseal) was maintained until the backfilling solution reached the micropipette tip by diffusion ( $\sim 40-90 \mathrm{~min})$ to minimize intracellular disruption by dialysis. When the concentration of the backfilling solution reached a sufficient concentration near the tip (estimated to be $\sim 0.2 \% \mathrm{NB}$ ), the plasma membrane patch was ruptured by mild suction and neurons were filled with NB by diffusion (filling time, $\sim 15-40 \mathrm{~min}$ ). In 10 cases, whole-cell configuration was achieved earlier than $40 \mathrm{~min}$ by spontaneous break-in. These cells stayed in whole-cell configuration longer (30-95 min) until sufficient NB concentrations were developed and resulted in dye coupling that was not significantly different from injections with shorter filling time (mean number of coupled neurons, $6.9 \pm 1.6$ ). Thus, prolonged intracellular dialysis in the whole-cell configuration does not appear to affect dye-coupling significantly.

$\mathrm{NB}$ is known to affect the intrinsic physiology of neurons ( $\mathrm{Xi}$ and $\mathrm{Xu}$, 1996; Schlösser et al., 1998), and we found that TRN neurons tended to be slightly depolarized during NB diffusion into soma. If neurons started spiking spontaneously, small negative currents were injected to silence them. DIC imaging of dye-filled neurons was used to help confirm the identity of the injected cell. To terminate an injection, the pipette was withdrawn carefully. Fluorescent images of dye-filled neurons were taken after pipette detachment at fixed light intensity and gain and used to estimate the LY concentration in the injected neurons later. Neurons that rapidly lost their LY signal after pipette detachment (less than $\sim 0.2 \%$ NB) were excluded from analysis. Slices containing a dye-filled neuron were incubated in the chamber for an additional $90 \mathrm{~min}$ to facilitate diffusion through GJ channels. Slices were then fixed in $4 \%$ paraformal- 
dehyde $>24$ h before additional histological processing. For comparisons of dye coupling between Cx36 KO and WT animals, the experimenters were blind to the genotypes of the mice during the injections and staining process.

The NB concentration in injected neurons was estimated from the fluorescence intensity of LY in the somata of the injected neurons measured from images taken right after pipette detachment, using NIH Image-J. In early experiments (all nine rat neurons and 14 of 43 mouse neurons), the somatic LY fluorescence was more than $\sim 0.2 \% \mathrm{NB}$ with only an approximate measurement of brightness range. In later experiments (29 of 43 mouse neurons), NB concentrations were estimated using measured LY signals. NB concentrations in TRN neurons ranged from $\sim 0.2$ to $0.8 \%$ (mostly from $\sim 0.3$ to $0.5 \%$ ) with an average $0.41 \%$. The estimated NB concentration was not correlated with the number of coupled neurons.

For control experiments, NB was injected into TRN neurons while they were bathed in GJ blockers, either carbenoxolone (CBX; 100-150 $\mu \mathrm{M})$ or mefloquine (MFQ; 50-75 $\mu \mathrm{M}$ ). Drug perfusion started after gigaseal formation. For CBX treatment, drug perfusion continued with an initial concentration $(100-150 \mu \mathrm{M})$ or with a slight reduced concentration of $70 \mu \mathrm{M}$ after $90 \mathrm{~min}$ to reduce toxic effects from long drug applications before slices were fixed. For MFQ treatment, drug perfusion started with 50-75 $\mu \mathrm{M}$ and then was reduced to $25 \mu \mathrm{M}$ after $30 \mathrm{~min}$ until slices were fixed. Mouse VB neurons (P12-P14) and hippocampal CA3 pyramidal neurons (P11-P13) were injected without any GJ blockers. For CA3 neurons, ionotropic glutamate receptors were blocked by APV (50$100 \mu \mathrm{M})$ and DNQX $(0-10 \mu \mathrm{M})$ to reduce spontaneous spiking activity. Estimated NB concentrations in all injected control neurons were more than $\sim 0.2 \%$.

Histology. Fixed slices were resectioned at 90 $\mu \mathrm{m}$ thickness, and the NB signal was developed via the $\mathrm{Ni}^{2+}$-enhanced $\mathrm{DAB}$ reaction. Sections were washed three times in phosphate buffer (PB; 0.1 m sodium phosphate, $\mathrm{pH}$ 7.4) before incubation in $0.5 \% \mathrm{H}_{2} \mathrm{O}_{2}$ for $>45 \mathrm{~min}$. Sections were rinsed three times in $\mathrm{PB}$, followed by incubation in $0.25 \%$ Triton X-100 in PBS (PB plus $0.15 \mathrm{M} \mathrm{NaCl}, \mathrm{pH} 7.4$ ) for $45 \mathrm{~min}$, and rinsed in $0.1 \%$ Tween 20/PBS for $5 \mathrm{~min}$. Sections were incubated overnight at $4{ }^{\circ} \mathrm{C}$ in avidin-biotinylated enzyme complex (Vector Laboratories) following the instructions of the manufacturer. For the $\mathrm{Ni}^{2+}$-enhanced $\mathrm{DAB}$ reaction, sections were preincubated in $0.05 \%$ $(\mathrm{w} / \mathrm{v}) \mathrm{DAB}$ and $0.3 \%(\mathrm{w} / \mathrm{v})$ nickel ammonium sulfate in Tris buffer $(0.05$ $\mathrm{M}$ Tris, $\mathrm{pH}$ 7.4) for $20 \mathrm{~min}$ and then transferred to the same solution containing $\mathrm{H}_{2} \mathrm{O}_{2}(0.006 \%)$. Color was developed for $2.25 \mathrm{~min}$ and stopped by rinsing in PB. Stained sections were dehydrated and coverslipped and then imaged using a Nikon E600 light microscope and a digital camera (SPOT; Diagnostic Instruments).

Two-dimensional (2D) drawings of injected neurons and coupled neurons were made with the aid of a camera lucida attachment for some dye-coupled neuronal clusters. To analyze axonal patterns of injected neurons, clusters that maintained relatively complete axon arbors were chosen. "Single axon-targeting" cells had arbors terminating in a single spot within VB, the posterior medial nucleus (POm), or the VB/POm border area. "Branched dual axon-targeting" cells had two separated arbors, one each in VB and POm. The border between the VB and POm
D

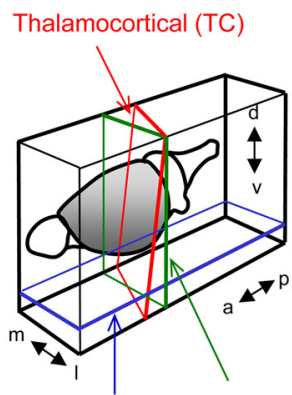

Horizontal $(\mathrm{Hz}) \quad$ Coronal
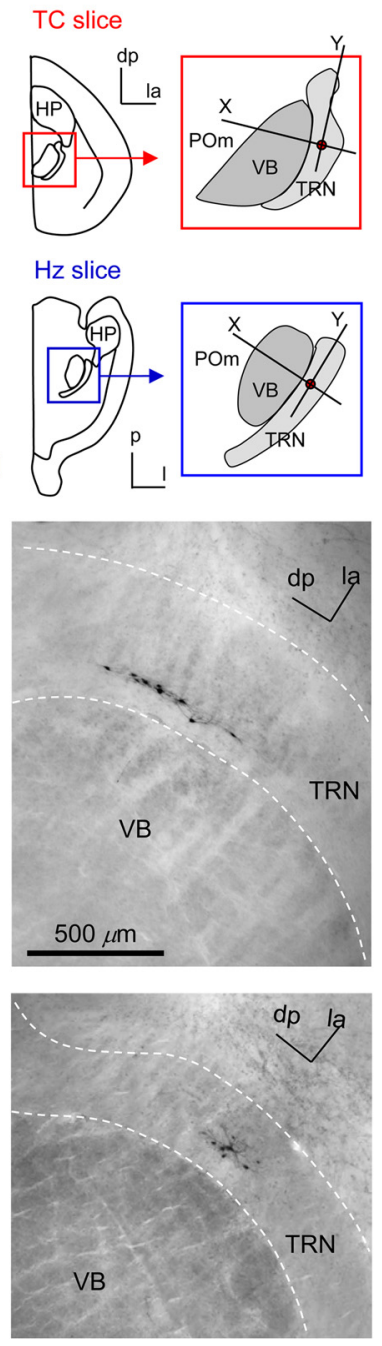

Figure 1. NB dye coupling of TRN neurons. $A$, Staining of cells, blood vessels, and extracellular matrix by NB. Image of a thalamic only with NB (0.5\%)-containing internal solution (no front-filling). Blue arrows indicate injected neurons. $\boldsymbol{B}$, An example of a is onster from an NB-injected TRN neuron from a rat TC slice. The blue arrow indicates the injected cell. Low magnifand $\mathrm{Hz}$ slices and the orientation of the TRN and VB in each slice. $x$ and $y$ axes were used for measuring coordinates of dye-coupled neurons. a, Anterior; d, dorsal; dp, dorsoposterior; HP, hippocampus; I, lateral; la, latero-anterior; m, medial; p, posterior; v, ventral.

was often identified by background staining from the $\mathrm{DAB}$ reaction (the $\mathrm{VB}$ is slightly darker than the POm). In the few cases when the VB/POm border was not evident from background staining, its position was estimated by the distance from the VB/TRN border. In the one case of a branched dual axon-targeting cell, the axon arborized in the VB and then extended some distance farther to the POm area but was cut before arborizing extensively in the POm.

Dye-positive cells were defined by these criteria: (1) the intracellular signal had a shape and size consistent with those of neuronal somata in TRN; and (2) the mean signal intensity (brightness) across the region of interest (soma area) was lower than three times the SD of background signals from a large neighboring area (i.e., a TRN area without coupled cells). Signal intensity was measured using NIH Image-J. For classification of the signal intensity of coupled neurons (see Fig. 3D), cells with brightness $\geq 70 \%$ and $<40 \%$ of background brightness were classified as "weak" and "strong," respectively. The rest of the cells were classified as "intermediate."

$3 D$ coordinates of neurons. 3D $(x, y, z)$ coordinates for all dye-coupled neurons were obtained from digital images (for $x, y$ coordinates) and by measuring the $z$-axis depth on microscope. The position of the dye- 
A
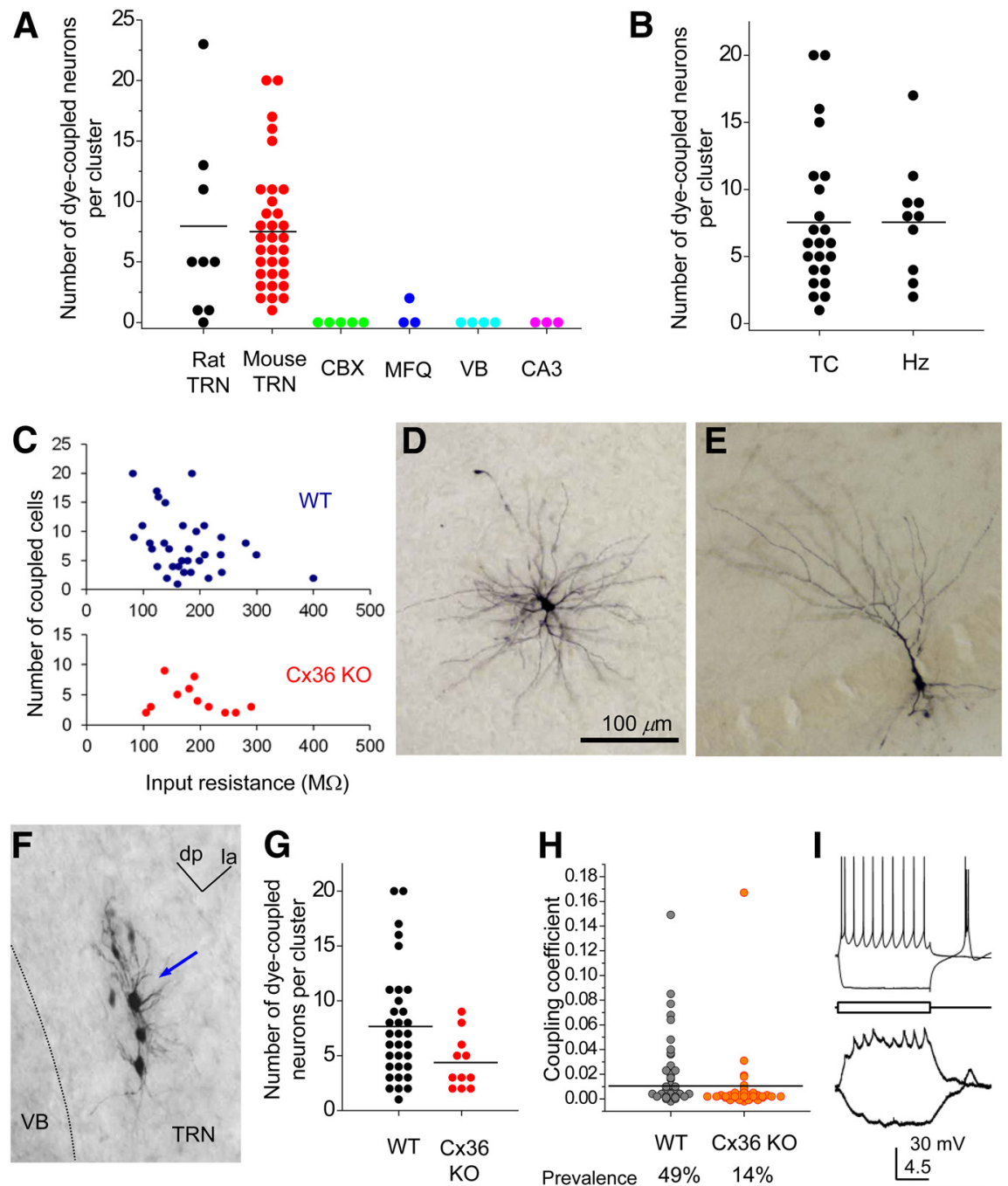

B

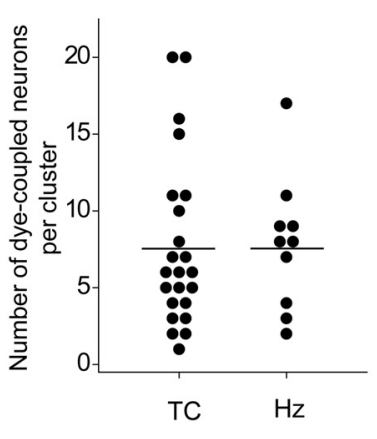

H

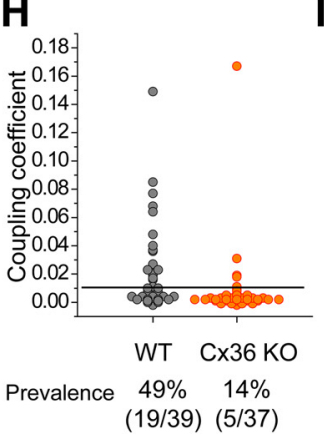

Figure 2. Prevalence of neuronal dye coupling. $A$, Numbers of dye-coupled neurons per injection: rat TRN neurons (control ACSF), mouse TRN neurons (control ACSF), CBX-treated (150-100 $\mu \mathrm{M}$ ) rat and mouse neurons, MFQ-treated (75-50 $\mu \mathrm{m}$ ) mouse neurons, mouse VB neurons (P12-P14, control ACSF), and mouse hippocampal CA3 pyramidal neurons (P11-P13) are shown. $\boldsymbol{B}$, Numbers of dye-coupled mouse neurons per cluster from slices cut in two different planes. C, Coupling in WT and Cx36 KO cells. Plot of input resistance of the dye-injected neuron versus the number of coupled neurons within each cluster. $\boldsymbol{D}$, NB-injected VB relay neuron (control ACSF). $E$, NB-injected hippocampal CA3 pyramidal neuron. $F$, An example of a dye-coupled cluster from a C 36 KO mouse. The blue arrow indicates injected neuron. dp, Dorsoposterior; la, latero-anterior. $\mathbf{G}$, Numbers of coupled neurons per cluster in the TRN of WT ( $n=32$ clusters) and Cx36 KO $(n=11)$ mice. $\boldsymbol{H}$, Plot of coupling coefficients measured by paired whole-cell recordings from TRN neuron pairs in WT and $\mathrm{C} \times 36 \mathrm{KO}$ mice. Parentheses indicate the number of coupled pairs/number of total measured pairs. $I$, An example recording from an electrically coupled TRN neuronal pair (coupling coefficient $=0.17$ ) from $(x 36 \mathrm{KO}$ mice. Voltage changes in a presynaptic neuron (top trace) induced by intracellular current steps (100 and $-150 \mathrm{pA}$ ) caused attenuated voltage changes in a postsynaptic neuron (bottom trace).

injected neuron was assigned as the 0 (reference) coordinate. The centers of the somata of individual coupled neurons were defined by their coordinates, and neurons were treated as points in space for additional analyses. In each slice from both the TC and Hz planes, the $x$-axis was defined as the line that passed through the center of the dye-injected neuron and ran perpendicular to the TRN/VB border and parallel to the section plane (Fig. 1D). The $y$-axis was defined as the line running through the primary neuron and parallel to the TRN/VB border and the section plane. The $z$-axis was defined as the line through the dye-injected neuron and perpendicular to the section plane. Shrinkage that occurred during dehydration along the $z$-axis was corrected by calculating the ratio of the measured thickness of final sections and the cutting thickness $(90 \mu \mathrm{m})$ before dehydration in each DAB staining run. Shrinkage in the $x-y$ plane was not corrected. The distance-dependent density of coupled neurons was calculated assuming that injected neurons were located near top of
I
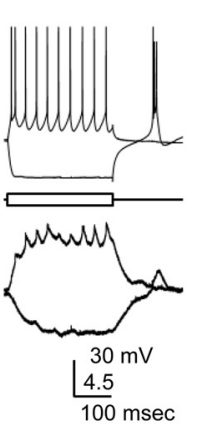

plate-like slices. The "maximum intersomatic distance" was the largest of all pairwise intersomatic distances in a cluster.

Cluster characterization using principal component analysis. After defining the $x, y, z$ coordinates of all neurons in a single dye-coupled cluster, principal component analysis (PCA) was performed to find the best-fitting axes describing the 3D distribution of neurons. Calculations were done with custom routines in MATLAB. The center of mass of all cell locations in a cluster was first calculated, and coordinates of all neurons were recalculated using this "center of mass" as the reference point. PCA generated three orthogonal axes. The first principal axis (PA1) indicates the direction along which the largest variance is generated. PA2 is the direction perpendicular to PA1 with the second largest variance, and PA3 is perpendicular to PA1 and PA2. The length of an axis is defined as $4 \times$ the SD of the spread of neurons along the axis (see Figs. 6-8). The $y-z$ plane was used to approximate the major plane of the TRN in both TC and $\mathrm{Hz}$ slices. The major plane of a cluster is defined by PA1 and PA2. Angles between PA1 or the major cluster plane and TRN plane were calculated using vectors defining the PAs.

Neuron cluster typing. Dye-coupled clusters of TRN neurons were divided into four main groups based on cluster size and geometry. First, if the number of neurons in a cluster was $<4$ or if the maximum intersomatic distance between neurons in a cluster was $<75 \mu \mathrm{m}$, that cluster was defined as a "small-sized" cluster. The rest of the clusters were grouped into three patterns-elongated, discoid, and sphericalaccording to a parameter based on the ratios of the lengths of their three PAs. $R_{1-2}$ is the ratio of the lengths of PA1 and PA2 $\left(R_{1-2}\right.$ is the length of $\mathrm{PA} 2 /$ length of PA1). $R_{1-3}$ is the ratio of the lengths of PA1 and PA3. For an ideal sphere, $R_{1-2}$ $=R_{1-3}=1$ (see Fig. $5 B$ ). For a thin discoid cluster, $R_{1-2}=1$ and $R_{1-3}$ is close to 0 . If both $R_{1-2}$ and $R_{1-3}$ are close to 0 , the ellipsoid becomes rod-shaped. Based on these properties, clusters with $R_{1-2}>0.7$ and $R_{1-3}>0.25$ were arbitrarily defined as "spherical" (see Fig. $5 A$ ). Clusters with $R_{1-2}>0.35$ and $R_{1-3}<0.25$ were defined as "discoid." Clusters with $R_{1-3}<R_{1-2}$ $<0.35$ were defined as "elongated."

Statistics. Student's $t$ tests were used unless otherwise specified. Data were expressed as the mean \pm SEM.

\section{Results}

\section{A novel dye-injection method}

The goal of our study was to reveal the spatial organization of GJ-coupled networks of TRN neurons. Dye coupling is the best method for this purpose. NB (molecular weight of $287 \mathrm{kDa}$ ) is a widely used tracer for dye-coupling studies because of its small size, biocompatibility, and good detection sensitivity. NB permeates GJ channels and produces robust dye coupling between GJcoupled neurons in the retina (Mills and Massey, 2000; Pan et al., 2010). However, early dye-coupling attempts in mammalian brain regions with LY (molecular weight of $443 \mathrm{kDa}$ ) or NB produced mixed results (Connors et al., 1983; Peinado et al., 1993; Benardo, 1997), and efforts with whole-cell pipettes using NB in 
electrically coupled GABAergic neurons mostly failed to demonstrate dye coupling (Gibson et al., 1999; Galarreta and Hestrin, 2001a; Landisman et al., 2002). Unsuccessful NB dye coupling was attributed to poor detection or impermeability of interneuron GJ channels to NB. To clarify whether NB can induce dye coupling between electrically coupled GABAergic neurons of the forebrain, we injected NB into TRN neurons in brain slices with a modified technique designed to minimize false-positive and false-negative outcomes.

A major technical problem has been that leakage of dye-containing internal solution during the micropipette approach stage can cause spurious staining of neurons. Recordings made with pipettes filled uniformly with $0.5 \% \mathrm{NB}$-containing solution produced a well stained central neuron (the neuron patched with the pipette) plus staining of many neighboring neurons, extracellular matrix, and blood vessels (Fig. 1A). The blood vessel staining was especially intense, possibly because of biotin transporters in blood vessel endothelial cells (Spector and Johanson, 2007). Similar patterns of background and neuronal staining were often obtained when the internal solution was deliberately pressure ejected into the extracellular space (data not shown). False-positive staining made it impossible to identify dye-coupling signals that were generated by intercellular diffusion through GJ channels.

To minimize spurious staining, we modified the method by adding a pipette front-filling technique, a fluorescent dye $\mathrm{LY}$, and a careful recording procedure (see Materials and Methods). During dye injection, LY served as an indicator of the local concentration of dye-containing solution within the tip of the micropipette, allowing us to form seals on the membrane before the backfilling solution reached a significant concentration at the tip and to inject relatively high and consistent concentrations of NB into neurons to minimize false-negative results (Lin et al., 2003).

\section{Dye coupling between GJ-coupled TRN neurons}

NB injection into TRN neurons using our modified method completely eliminated staining of blood vessels and extracellular matrix staining. It also resulted in staining of multiple neighboring neurons in addition to those injected (see Figs. $1 B, C, 6 A, 7 A$, $8 A)$. Most dye-injected neurons were easily recognized by their strong signal intensities and well stained dendrites and axons. Dye-coupled neuronal somata were often found among the dendritic arbors of the injected neuron. Figure $1, B$ and $C$, shows two examples of dye-coupled clusters from rat TRN. In Figure $1 B$, the cluster has a strikingly elongated shape $\sim 300 \mu \mathrm{m}$ long, running parallel to the long axis of the TRN (for similar arrangements, see Figs. $6 A, 7 A$ ). In contrast, Figure $1 C$ shows a dye-coupled cluster
B
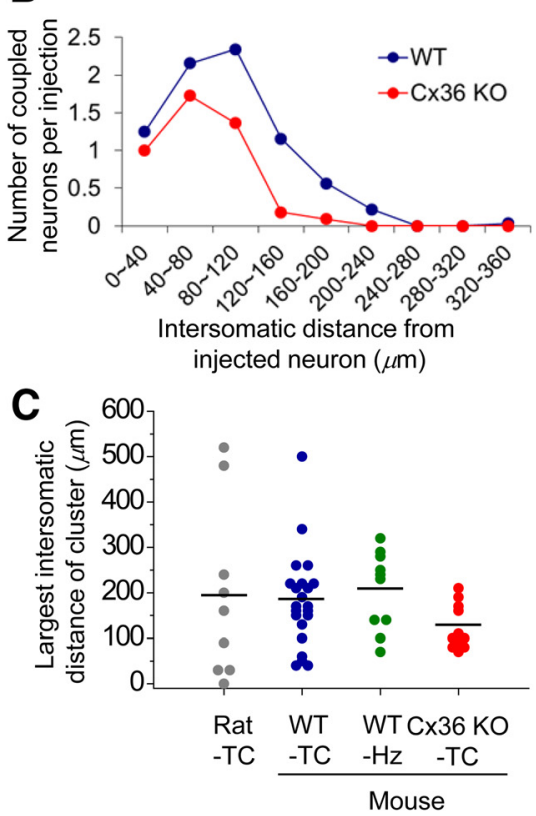

E

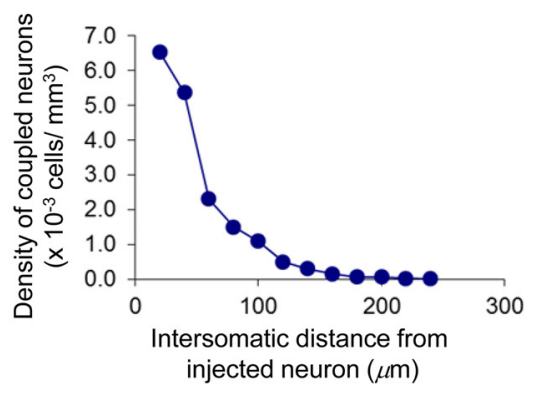

Figure 3. Distances between coupled neurons. $\boldsymbol{A}$, Histograms of intersomatic distances between injected TRN neurons and dye-coupled TRN neurons from all NB injections. Rat-TC (gray), Rat TRN neurons in TC slices; WT-TC (blue), WT mouse TRN neurons TC slices; WT-Hz (green), WT mouse TRN neurons in Hz slices; Cx36 K0-TC (red), Cx36 K0 mouse TRN neurons in TC slices. The

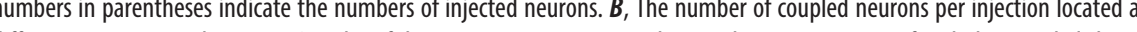
respectively. $\boldsymbol{E}$, Density of dye-coupled neurons from a WT mouse TRN at different intersomatic distances. The number of coupled neurons was divided by the volume of tissue area within the indicated distance ranges from injected neurons.

with neurons that spread more radially across the long and short axes of the TRN.

To investigate the spatial structure of GJ-coupled clusters, a total of nine TRN neurons from rats and 33 TRN neurons from WT mice were injected with NB using the injection method described above. The mean number of neurons per cluster (including the dye-injected neuron) was $9 \pm 2.5$ neurons (range, 1-24) from rat and $8.7 \pm 0.9$ neurons (range, 2-21) from mouse (Fig. $2 A)$. In the mouse TRN, injections were performed in slice preparations cut at two approximately perpendicular angles: the TC (Agmon and Connors, 1991) and Hz (Fig. 1D) planes. Dyecoupled clusters of very similar size were obtained from each plane (TC, $8.7 \pm 1.2 ; \mathrm{Hz}, 8.8 \pm 1.4 ; p>0.9 ;$ Fig. $2 B$ ), indicating no significant effect of slicing angle. The size of dye-coupled clusters was negatively correlated with the input resistances of the injected neurons measured during dye injection (Fig. 2C; WT, $p<0.025$ ), which is consistent with gap junctional conductances 
A

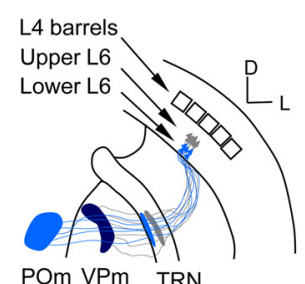

POm VPm TRN
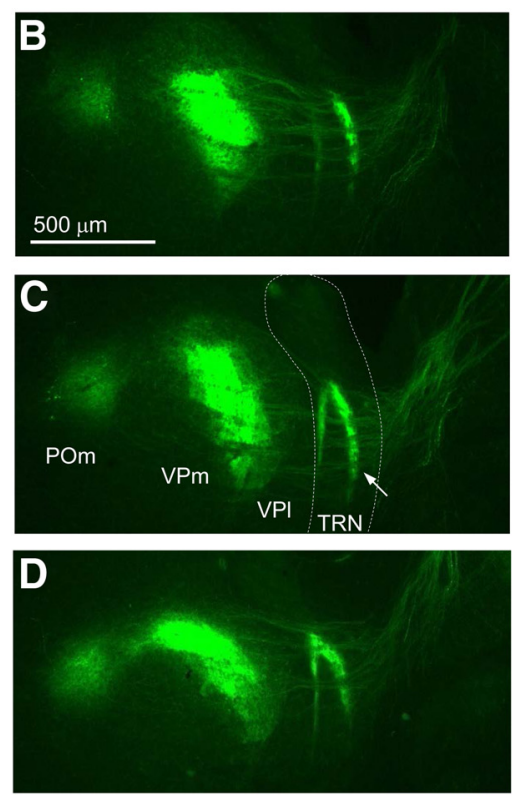
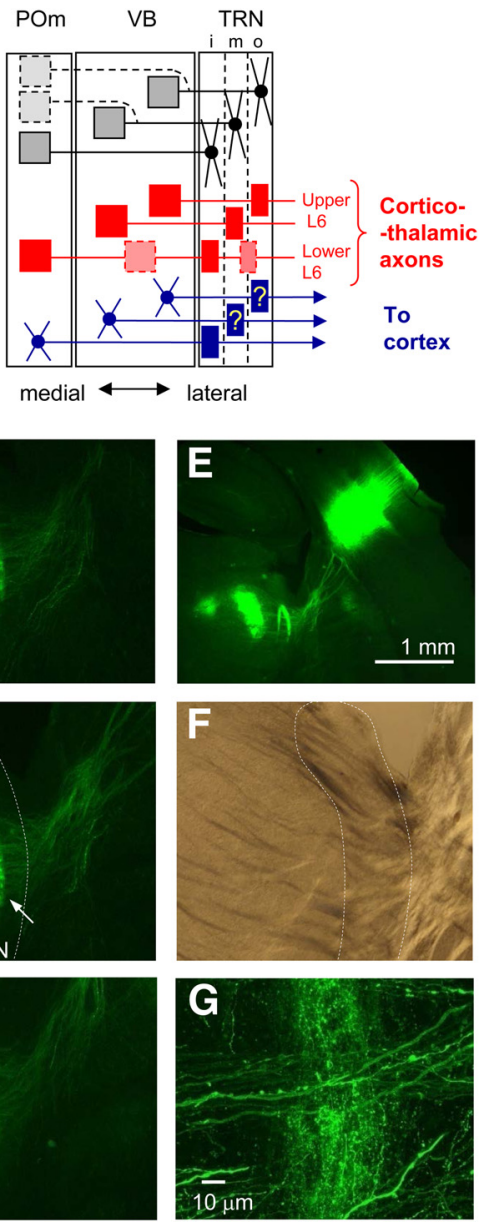

Figure 4. Spatial patterns of axonal arbors in the thalamus from deep-layer CT neurons of the primary somatosensory (S1) cortex. $A$, Left, Schematic demonstrating connectivity of L6CT neurons of a single-barrel column of S1 cortex. Upper L6 neurons (gray) project to the VB, forming thin discoid axonal arbors in the intermediate/outer tier of the TRN. Lower L6 neurons (light blue) project to the VB and POm, sending collaterals to form axonal arbors mainly in the inner tier of the TRN. The dark blue terminals in the VB indicate coextensive terminals of upper and lower L6 neurons (modified from Bourassa et al., 1995). Right, Diagram of the proposed organization of the somatosensory sector of the TRN. Filled circles and boxes indicate somata and axon terminals, respectively. Lines indicate axons or dendrites. Dotted boxes and lines indicate occasionally observed projections. i, Inner tier; m, middle tier; 0, outer tier. Axonal projection patterns of VB neurons to the TRN (question marks) are not clearly reported. This diagram is mainly based on previous anatomical studies (Crabtree, 1992; Bourassa et al., 1995; Pinault et al., 1995). $\boldsymbol{B}-\boldsymbol{D}$, Fluorescent images of three serial TC slices containing EYFPexpressing $\mathrm{CT}$ fibers originating from a single small injection of virus into the deep S1 cortex. White dotted line in $\boldsymbol{C}$ indicates the TRN border. $\boldsymbol{E}$, Low-magnification fluorescent image of the slice containing the area shown in $\boldsymbol{C}$. $\boldsymbol{F}$, Bright-field image of the TRN area of $\boldsymbol{C}$. $\boldsymbol{G}$, Highmagnification image of the TRN area marked by the white arrow in $\boldsymbol{C}$. VPm, Ventral posterior medial nucleus; VPI, ventral posterior lateral nucleus.

contributing significantly to the input conductance of each neuron (Deans et al., 2001; Amitai et al., 2002; Hjorth et al., 2009). Our data indicate that GJ-coupled clusters of TRN neurons are heterogeneous in size and that single TRN cells can be connected to $>20$ other neurons.

Several control experiments confirmed that false-positive staining was rare under these conditions. First, in all cases, LY stained only the injected cell and did not appear in neighboring cells. This is consistent with reports that Cx36-containing GJ channels are very poorly permeable to LY (Bukauskas, 2012). Second, GJ blockers, either CBX or MFQ (Rozental et al., 2001; Cruikshank et al., 2004) nearly eliminated dye coupling between

A

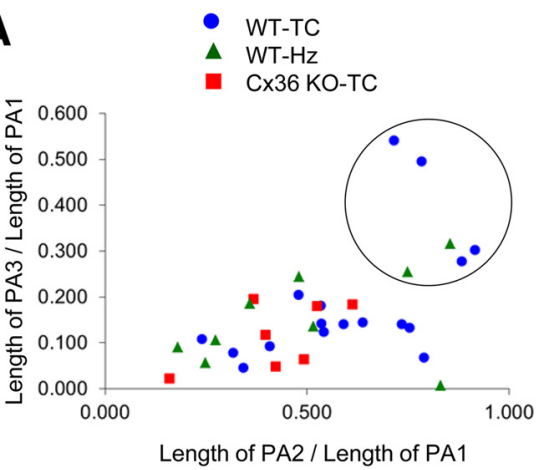

B

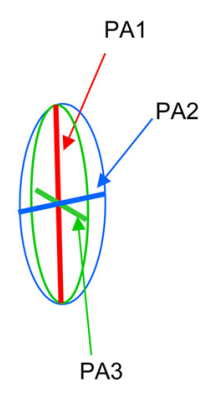

Figure 5. Classification of cluster patterns. $A$, Plot of $R_{1-2}$ versus $R_{1-3}$ (see Materials and Methods) of all coupled clusters. Classification of cluster pattern was done based on this plot. Clusters within the black circle are spherical clusters. Small-sized clusters are not included in this plot. $\boldsymbol{B}$, Schematic showing PA1, PA2, and PA3 defining an ellipsoid.

TRN neurons (Fig. 2A). Third, NB did not lead to dye coupling in neurons that are not expected to be GJ coupled. Mouse thalamic relay neurons in the VB nucleus are not electrically coupled at P12 (Lee et al., 2010), and NB injection produced only singleneuron labeling (Fig. 2A,D). NB injections into hippocampal CA3 pyramidal neurons from P11-13 mice also labeled only single cells (Fig. 2A,E). These results suggest that dye coupling among TRN cells is not attributable to spurious staining from extracellular dye, but very likely NB diffused through specific intercellular channels from the injected neurons.

NB intensity in dye-coupled neurons appeared to be consistently lower than the intensity of directly injected neurons (Figs. $1 B, C, 6 A, 7 A, 8 A)$. Also, $\mathrm{NB}$ staining of axons from the coupled neurons was usually too weak to be traced clearly; in contrast, axons from injected neurons were usually strongly labeled. Previous paired recordings showed that the junctional conductances between coupled TRN cell pairs ranged from undetectably low to as high as $2 \mathrm{nS}$ (Parker et al., 2009). It appears that NB diffusion through coupled TRN networks is relatively slow, so that NB concentrations cannot equilibrate during the incubation period even among cell pairs with high junctional conductances. This implies that the majority of detectable NB coupling is from firstorder GJ connections.

\section{Spatial dimensions of GJ coupling in TRN}

The intersomatic distances between injected neurons and their dye-coupled cohorts ranged from closely adjacent to as far as 340 $\mu \mathrm{m}$. Figure $3 A$ plots the intersomatic distances from all analyzed clusters: for clusters in rat TC slices, it was $115 \pm 10 \mu \mathrm{m}$, and for WT mouse TRN, it was $96 \pm 4$ and $88 \pm 6 \mu \mathrm{m}$ from TC and $\mathrm{Hz}$ slices, respectively (Fig. $3 A, B$ ). To estimate the overall size of each dye-coupled cluster, the maximum intersomatic distances among neurons in individual clusters were measured. The mean maximum distances were 1.7-2.3 times the mean intersomatic distances; $193 \pm 63 \mu \mathrm{m}$ for rat TC slices, $181 \pm 23 \mu \mathrm{m}$ for mouse TC slices, and $204 \pm 27 \mu \mathrm{m}$ for mouse Hz slices (Fig. $3 C$ ). For the majority of clusters, the maximum intersomatic distances are a measure of their extent within the plane of TRN.

The NB staining intensity of dye-coupled neurons varied widely. We categorized the intensity of each neuron in a cluster according to a 3-point scale. Among coupled neurons, the general staining intensity decreased with intersomatic distance within the cluster (Fig. 3D). Nevertheless, some intensely stained neurons were observed even at long distances $(>160 \mu \mathrm{m})$, suggesting that strong GJ coupling can occur between some widely 
spaced neurons. It should be noted that the density of somata in a dye-coupled cluster is considerably lower than the local density of all neurons. The probability of coupling, calculated from the ratio of coupled/total neurons, decreased rapidly with distance from the injected cell (Fig. 3E). This decrease is partly attributable to directional bias of coupling, which is discussed below. We could consistently find uncoupled somata interspersed among dye-coupled somata, indicating that coupling does not depend on proximity from the injected neuron alone.

\section{Cx36 and GJ coupling}

$\mathrm{Cx} 36$ is thought to be a major constituent of neuronal GJ channels in the mammalian brain (Connors and Long, 2004), including TRN (Landisman et al., 2002). Surprisingly, when TRN neurons from Cx36 KO mice were injected with NB, dye coupling was reduced but not abolished (Fig. 2F,G). The number of coupled neurons per cluster was significantly lower in KO tissue than in TRN from WT mice (WT, $7.7 \pm 0.9$; Cx36 KO, $4.4 \pm 0.7 ; p<$ 0.05 ; Fig. $2 G$ ). The spatial dimensions of clusters (intersomatic distances of WT, $93.3 \pm 3.3 \mu \mathrm{m} ; \mathrm{KO}, 68.9 \pm 5.0 \mu \mathrm{m} ; p<$ 0.05 ; Fig. $3 A, B)$ and the maximum intersomatic distance (WT, $188 \pm 18 \mu \mathrm{m}$; KO, $124 \pm 15 \mu \mathrm{m} ; p<0.02$; Fig. $3 C$ ) were also significantly smaller in $\mathrm{Cx} 36 \mathrm{KO}$ mice than WT. Interestingly, the largest clusters (maximum intersomatic distance $>220$ $\mu \mathrm{m})$ were selectively absent in the Cx36 $\mathrm{KO}$, whereas they comprised $\sim 35 \%$ of clusters in WT slices (Fig. 3C). The data suggest that $\mathrm{Cx} 36$ is not necessary for all interneuronal coupling in the TRN and that $\mathrm{Cx} 36$ is particularly critical for longdistance GJ coupling.

A previous electrophysiological study from our laboratory found that the prevalence of electrical coupling was very low among TRN neurons from Cx36 KO mice (WT, 32\%; KO, $\sim 3 \%$; Landisman et al., 2002). Here we performed additional paired whole-cell recordings from TRN neurons of WT and Cx36 $\mathrm{KO}$ animals. The strong difference between WT and KO coupling remained, but the prevalence of electrical synapses for both genotypes was higher than in the previous study (WT, $49 \%$; Cx36 KO, $14 \% ; p<0.001, \chi^{2}$ test; Fig. $\left.2 H, I\right)$. The coupling coefficient was weaker between KO pairs than WT pairs when comparing all tested pairs ( $p<0.025$, Mann-Whitney test). There was one very strongly coupled pair in the KO group; the coupling coefficients of WT and KO pairs were not different when comparing only the coupled pairs. The persistence of both dye coupling and electrical coupling between some TRN neurons in Cx36 KO mice implies that unknown GJ-forming proteins are expressed in TRN neurons. There were no signs of LY dye coupling in either WT or KO cells, suggesting that both Cx36- and non-Cx36-dependent GJ channels have limited permeability to that anionic tracer.
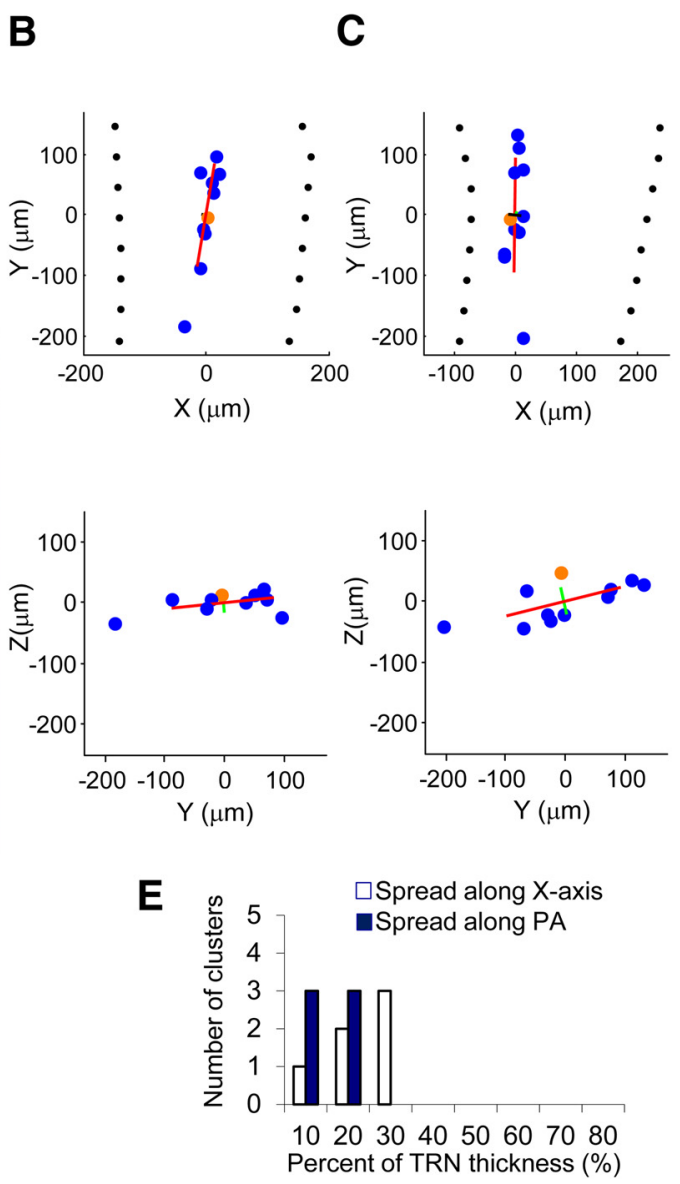

Figure 6. Elongated dye-coupled clusters from mouse TRN. A, An example of an elongated cluster from a mouse Hz slice. Top, Low-magnification image of the cluster. p, Posterior; I, lateral. Bottom, High-magnification image of the same cluster. The blue arrow indicates the injected neuron. Red lines indicate the directions of the $x$ and $y$ axes used for analysis. $\boldsymbol{B}$, Plot of the locations of plane. Bottom, $y-z$ plane. Red, green, and black lines indicate PA1, PA2, and PA3, respectively. PA2 and PA3 of this elongated cluster are difficult to see because of their short length. The lengths of the PAs are $2 \times$ SD along the axes. C, Plot of the locations of the TRN plane ( $y-z$ plane) of all elongated clusters from WT mice $(n=6)$. . Histogram of portions occupied by clusters ( $95 \%$ spread) with respect to the whole thickness of the TRN. Blank bars indicate the values calculated from spread along the $x$-axis. Filled bars indicate the values calculated from spread along one of PAs that was closest to the $x$-axis.

\section{Two spatial patterns of GJ coupling in TRN}

GJ-coupled neuronal networks are often assumed to be isotropic and spatially homogenous (Amitai et al., 2002; Hjorth et al., 2009; Vervaeke et al., 2010). However, many brain regions have a nonhomogeneous organization and discrete domains, and the thalamus is no exception. For example, the somatosensory VB complex has neuronal clusters, called barreloids, devoted to information processing of vibrissae and body surfaces; the adjacent higher-order region, the POm, is more extended and diffuse. The TRN is topographically organized, reflecting the arrangement of its connections with the thalamic relay nuclei; neurons of the inner tier of TRN may be exclusively innervated by axons from POm relay neurons and collaterals of POmprojecting corticothalamic (CT) fibers from lower layer 6 (L6; Bourassa et al., 1995; Deschênes et al., 1998; Wimmer et al., 2010; Fig. $4 A$ ). Middle and outer tiers of the TRN are mainly connected to the $\mathrm{VB}$ and its $\mathrm{CT}$ afferents from upper L6. In general, the TRN contains thin slab-like functional domains corresponding to body regions that are aligned parallel to the TRN plane and organized topographically, mirroring connections with the VB and POm (Shosaku et al., 1984; Bourassa et al., 1995; Pinault et al., 
A
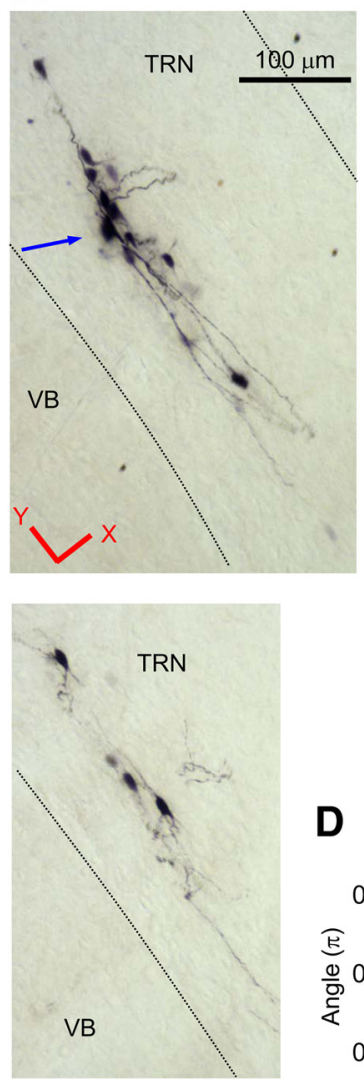

B

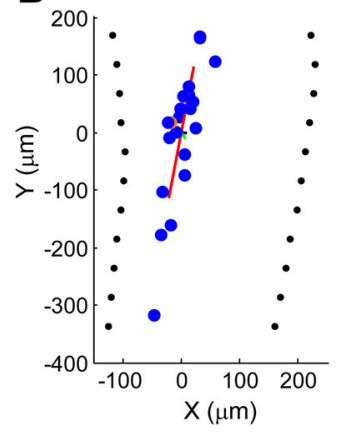

C
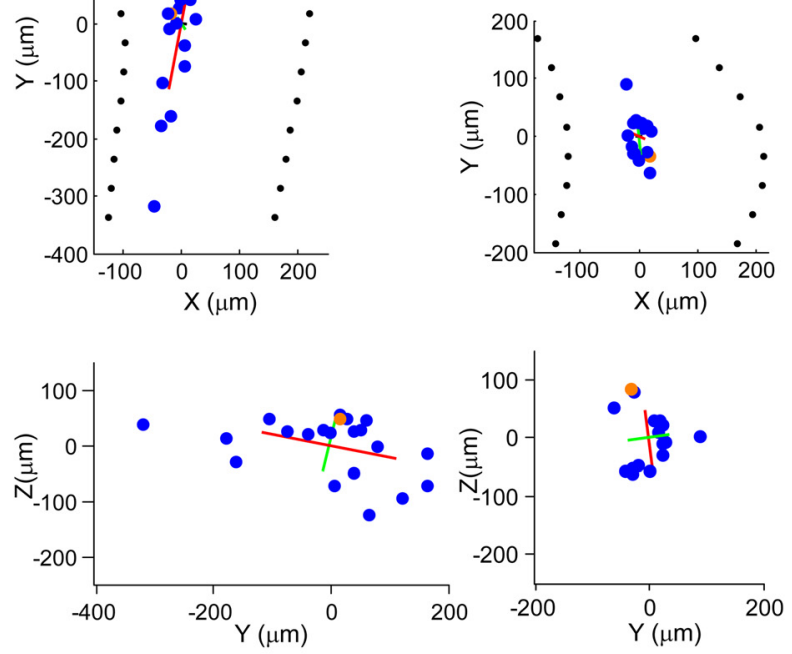

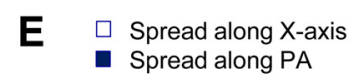

Spread along PA

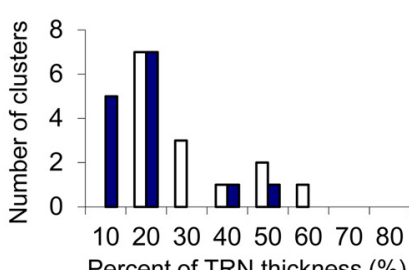

Percent of TRN thickness (\%)

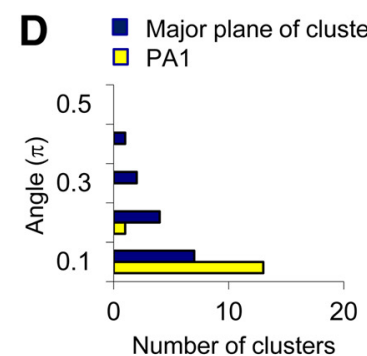

Figure 7. Discoid dye-coupled clusters from mouse TRN. A, An example of a discoid cluster from a mouse TC slice. Top, Image of the top part of the cluster (the first section from resectioned slice). Bottom, Image of the lower part of the cluster (the second section). The blue arrow indicates the injected neuron. Red lines indicate directions of the $x$ and $y$ axes used for analysis. $\boldsymbol{B}$, Plot of the locations of neurons in the cluster in $A$. Blue and orange dots represent dye-coupled neurons and an injected neuron, respectively. Top, $x-y$ plane. Bottom, $y-z$ plane. Red, green, and black lines indicate PA1, PA2, and PA3, respectively. PA3 of discoid clusters are difficult to see because of their short lengths. The lengths of the PAs are $2 \times S D$ along the axes. $C$, Plot of the locations of neurons from another example of discoid clusters. $\boldsymbol{D}$, Histogram of angles between PA1 or major plane (defined by PA1 and PA2) of clusters and the TRN plane ( $y-z$ plane) of all discoid clusters from a WT mouse TRN $(n=14)$. Yellow bars, Angles between the PA1 and TRN plane. Blue bars, Angles between the major plane of the cluster (PA1-PA2) and the TRN plane. E, Histogram of portions occupied by clusters ( $95 \%$ spread) with respect to the whole thickness of TRN. White bars, Values calculated from the spread of neurons in a cluster along the $x$-axis. Blue bars, Values calculated from the spread of neurons along the PA of one of the clusters, which was closest to the $x$-axis.

1995). We tested whether this spatial organization of the TRN has any relationship to the patterns of its GJ-coupled clusters of neurons.

To highlight this organization in the mouse, we injected enhanced yellow fluorescent protein (EYFP)-encoding lentiviruses into single loci of mouse barrel cortex to examine CT axon terminals within the TRN. In all three cases in which injections transduced deep-layer neurons of approximately four to nine barrel columns, CT collaterals arborized densely within two thin slabs of TRN, one around the middle of its thickness and a second deeper and adjacent to the TRN border with the VB (Fig. 4B-E). These slabs of labeling occupied only a small portion of the TRN thickness but spread widely along its major plane. High magnification often showed collaterals of single CT axons traveling long distances within the TRN (Fig. 4G). This organization is consistent with previous proposals for a laminated functional organization of rat and rabbit TRN with specific connections to thalamic relay nuclei (Shosaku et al., 1984; Crabtree, 1992;
Bourassa et al., 1995; Pinault et al., 1995; Lam and Sherman, 2007).

We observed that the patterns of dyecoupled TRN neurons are strikingly biased in orientation and shape. Most clusters had a thin, elongated distribution of coupled neurons running parallel to the long axis of the TRN (Figs. $1 B, 6 A, 7 A$, $9 A-C)$. To characterize the shapes and orientations of the clusters more systematically, we performed a $3 \mathrm{D}$ analysis of neuron positions and then applied PCA to define the major principal axis (PA1) and two additional orthogonal axes (PA2, PA3) (for details, see Materials and Methods). Based on the relative spread along three the PAs, the shapes of dye-coupled clusters were divided into three categories: (1) elongated; (2) discoid; and (3) spherical (Fig. 5). Small clusters (fewer than four neurons, or a maximum intersomatic distance of $<75 \mu \mathrm{m}$ ) were excluded from the PCA because it was difficult to define patterns for them. The incidence of small clusters were 23\% (5 of 22) in WT TC slices, $10 \%$ (1 of 10) in WT $\mathrm{Hz}$ slices, and 36\% (4 of 11) in Cx36 KO TC slices.

The coupled neurons of elongated clusters spread mainly along PA1 and very narrowly along PA2 and PA3 (Fig. $6 A)$. For all elongated clusters, PA1 ran closely parallel to the VB/TRN border (Fig. 6B-D). Along the short axis of the TRN, or the PA closest to the short axis of the TRN, neurons spread $10-30 \%$ of the thickness of the TRN (Fig. 6B, C,E). The incidences of elongated clusters were $14 \%$ (3 of 22) in WT TC slices, 30\% (3 of 10) in WT Hz slices, and 9\% (1 of 11) in Cx36 KO TC slices.

The coupled neurons of discoid clusters spread extensively along PA1 and PA2, but were distributed very narrowly along PA3 (Fig. 7A). PA1 and PA2 ran closely parallel to the $\mathrm{VB} / \mathrm{TRN}$ border plane, forming thin disks embedded in the plane of the TRN (Fig. $7 B-D$ ). Only a few of the discoid clusters (3 of 14) deviated much $(0.3-0.4 \pi)$ from the TRN plane (Fig. $7 D)$, probably because of possible deviation of slicing angle from the perpendicular angle to the TRN plane. The spread of neurons along the short axis of the TRN occupied $\sim 30 \%$ of its thickness (Fig. $7 B, C, E$ ). When the thickness along the PA closest to the short axis of the TRN was considered, most discoid clusters occupied $<20 \%$ of the TRN thickness (Fig. $7 E$ ). The incidences of discoid clusters were 45\% (10 of 22) in WT TC slices, $40 \%$ (4 of 10 ) in WT Hz slices, and 55\% (6 of 11) in Cx36 KO TC slices.

Elongated and discoid clusters together form the majority (63\%) of coupled clusters of the TRN. Their shapes and orientations are strikingly similar with those of the proposed functional domains of the TRN (Fig. 4), suggesting that GJs in these clusters preferentially connect neurons within the same domains or a substructure of them. Although these clusters are mostly embed- 
ded in the TRN plane, the orientations of PA1s varied from vertical to horizontal.

The coupled neurons of spherical clusters spread far along all three PAs (Fig. $8 A-D)$. No single plane of orientation was evident, unlike the elongated or discoid clusters. Because of the thinness of the TRN, spherical clusters occupied a larger portion-approximately $45 \%$ of the thickness of the TRN-than the other cluster types (Fig. 8E). In fact, 50\% (three of six) of the spherical clusters occupied a very large portion $(60-70 \%)$ of the thickness (Fig. $8 B, C, E$ ). The incidences of spherical clusters were $18 \%$ (4 of 22) in WT TC slices, $20 \%$ (2 of 10) in WT Hz slices, and $0 \%$ (0 of 11) in Cx36 KO TC slices. The spread of coupled neurons across multiple tiers of the TRN suggests that GJs in spherical clusters connect neurons in multiple functional domains of the TRN, in contrast to the elongated and discoid groups.

\section{Differential axonal projections of dye-coupled clusters}

We looked for correlations between the spatial patterns of dye-coupled clusters and other features of the TRN neurons they comprised. The relative position of the injected neurons in the TRN did not obviously predict the number of coupled neurons or the spatial pattern of clusters, except that the injected neurons with spherical clusters tended to be located in the middle tier of the TRN.

Axons of injected TRN neurons terminating in the relay nuclei were well preserved in many injections from TC slices $(41 \%, 9$ of 22 ) but not from $\mathrm{Hz}$ slices ( 0 of 10). Six neurons from WT mouse TRN sent axons to single discrete regions in the $\mathrm{VB}, \mathrm{POm}$, or the VB/POm border area (Fig. $9 A-C$ ). The remaining three neurons sent branched axons with terminals in both the VB and POm (Figs. 8A, 9D). Consistent with the laminated organization of the TRN (Pinault et al., 1995; Fig. 4A), the position of the TRN neurons was topographically aligned with their projection to the POm or VB (Fig. 9A-D). There was a striking correlation between axonal projection patterns and the spread of dye-coupled clusters of neurons along the short axis of the TRN. All three injected TRN neurons with branched axons terminating in both the POm and VB had spherical clusters, with a broad spread of neurons along the thickness of the TRN (Fig. 8A, $9 D, E)$, whereas the six neurons that projected to single regions in the VB or POm had small, elongated, or discoid clusters narrowly spread across the thickness of the TRN (Fig. 9A-C,E; Fisher's exact test, $p<0.02$ ). This indicates that TRN neurons forming spherical clusters across multiple tiers of the TRN tend to make dual projections to both the first- and higher-order thalamus; these constitute a group distinct from neurons forming elongated or discoid clusters that send a single localized projection (Fig. $9 F)$. Spatial patterns and axonal projections together suggest that
B C
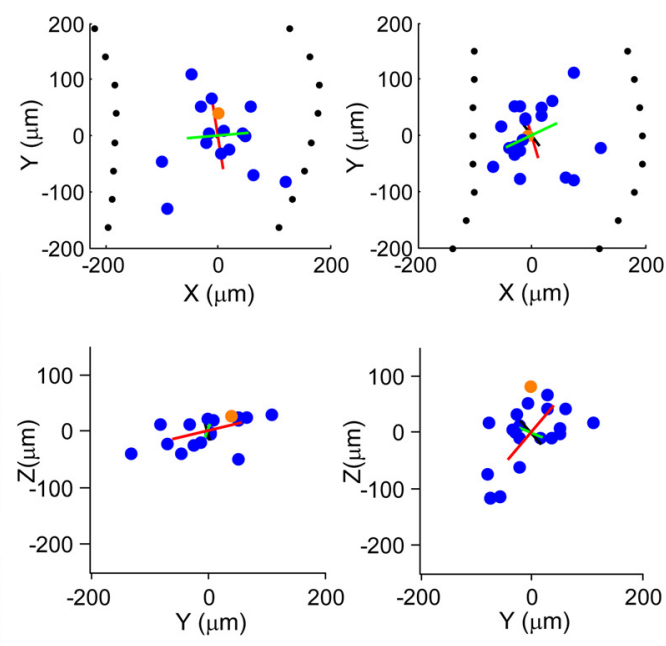

E

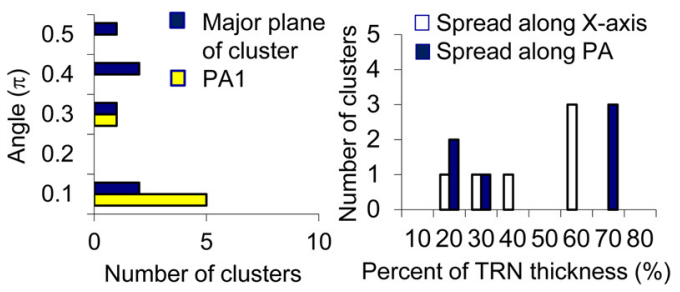

Figure 8. Spherical dye-coupled clusters from mouse TRN. A, An example of a spherical cluster from a mouse TC slice. Top, Camera lucida drawing of the cluster in low magnification. Middle, Low-magnification image of the cluster. Black arrows indicate axon terminals from an injected neuron. dp, Dorsoposterior; la, latero-anterior. Bottom, High-magnification image of the same

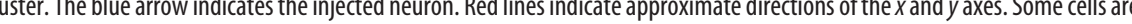
lurry because they are out of the focal plane. $\boldsymbol{B}$, Plot of the locations of neurons in the cluster in $\boldsymbol{A}$. Blue and orange dots represent neurons from another example of spherical clusters. $D$. Histogram of angles between PA1 or major plane of clusters and the (he by clusters ( $95 \%$ spread) with respect to the whole thickness of TRN. White bars, Values calculated from the spread of neurons in a cluster along the $x$-axis. Blue bars, Values calculated from the spread of neurons along the PA of one of the clusters, which was closest to the $x$-axis.

spherical clusters synaptically connect neurons between multiple domains of TRN and relay nuclei, whereas elongated or discoid clusters connect neurons within single domains of TRN and relay nuclei.

\section{Discussion}

The spatial organization of GJ-coupled GABAergic networks may play a critical role in determining how correlated inhibition is spatially integrated and distributed within a neural circuit. We have developed a sensitive dye-coupling method that induces NB staining among the GJ-coupled GABAergic neurons of the TRN. Staining patterns showed that GJ coupling occurs selectively, forming two distinct networks of inhibitory neurons with different geometric and axonal projection patterns. Their characteristics strongly suggest that TRN cell clusters have distinct functions that may underlie a variety of thalamic circuit operating modes.

\section{Architecture of GJ-coupled TRN neuronal networks}

Neurons in the somatosensory sector of the TRN are topographically organized, reflecting the arrangement of its connections with the thalamic relay nuclei (Crabtree, 1992; Bourassa et al., 
A

B
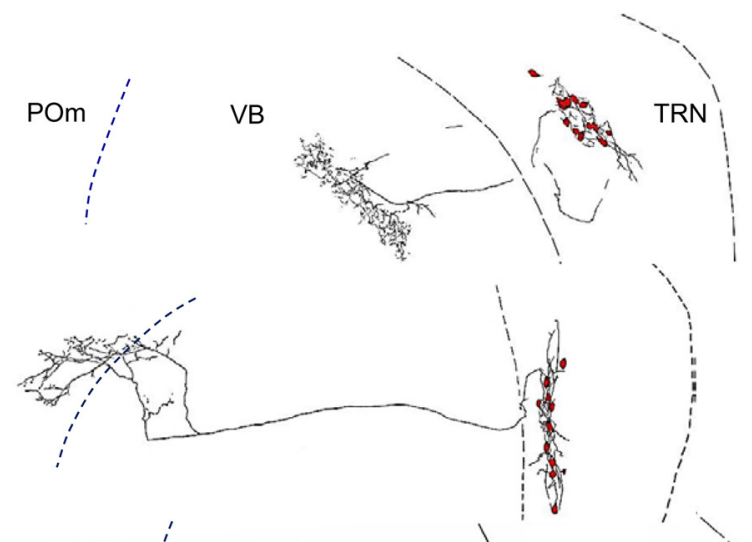

C

D
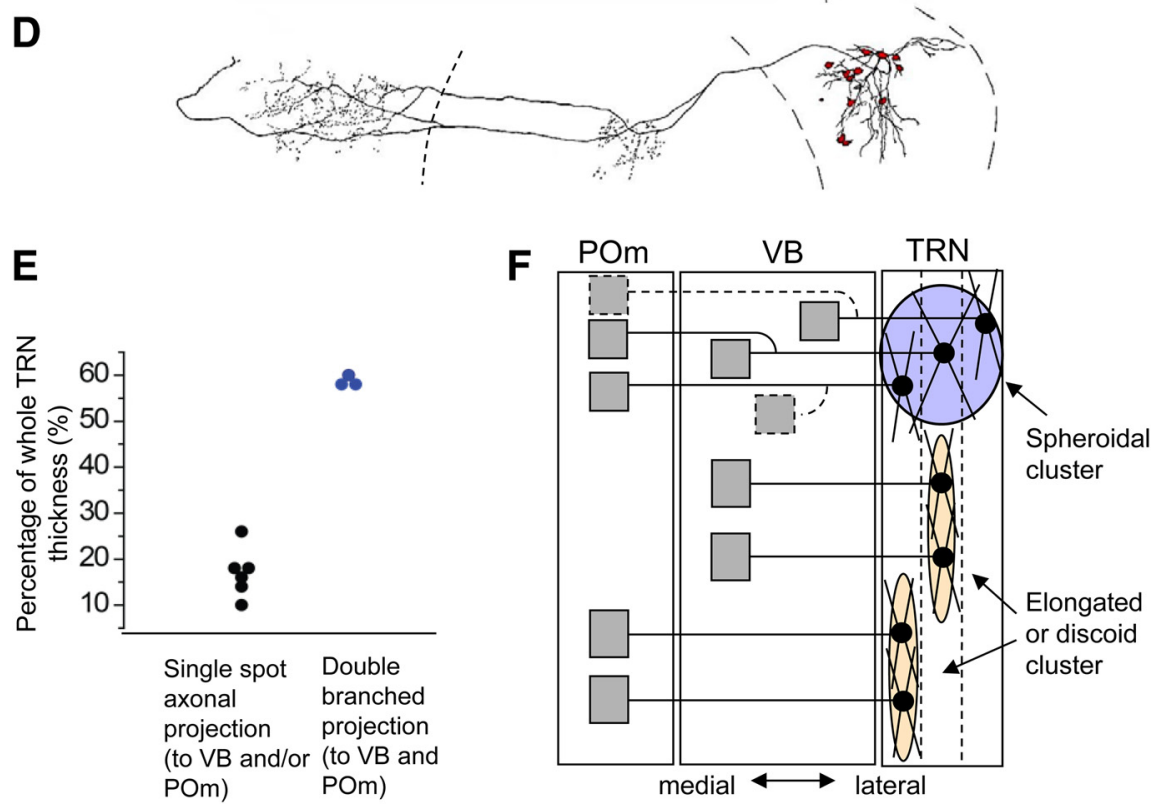

Figure 9. Axonal projections and dye-coupling patterns. $\boldsymbol{A}$, Drawing of an example of a coupled cluster and its injected TRN neuron sending axon terminations to the VB. The dashed line in the thalamus indicates the approximate location of the VB/POm border. $\boldsymbol{B}$, Drawing of a coupled cluster and its injected TRN neuron sending axon terminals to the VB/POm border area. $\boldsymbol{C}$, Drawing of a coupled cluster and its injected neuron sending axon terminations to the POm. D, Drawing of a coupled cluster and its injected TRN neuron sending branched axon terminations to both the VB and POm. $\boldsymbol{E}$, Spread of clusters along the short axis of the TRN versus the axonal projection pattern. $\boldsymbol{F}$, Schematic diagram of distinct GJ-coupling connectivity patterns. The blue circle indicates the connectivity pattern of spherical clusters, and the yellow ellipses indicate connectivity patterns of elongated or discoid clusters. Black circles and gray boxes indicate somata and axon terminals, respectively. Lines indicate axons or dendrites. Axons with dashed lines in the spherical cluster indicate uncertainty whether neurons in this cluster type are exclusively cells with branched axons or mixed populations with branched and nonbranched axons.

1995; Pinault et al., 1995). The TRN contains thin slab-like domains corresponding to body regions that are aligned parallel to the TRN plane. The majority of dye-coupled clusters in the TRN formed elongated or discoid patterns, aligned along the TRN plane and occupying only a small portion of its thickness. The similarity of shape, orientation, and size of these clusters to proposed functional domains of the TRN strongly suggests that GJ coupling of TRN neurons is not random but rather preferentially connects functionally related neurons in a thin tier of the TRN.
These clusters range in shape and size from discoid clusters that have similar shapes with functional TRN domains for a single barreloid, to extremely sharp elongated clusters. This diversity may derive from complex substructures of functional domains in somatosensory thalamus, as demonstrated in the rat whisker system (Pierret et al., 2000; Desîlets-Roy et al., 2002; Urbain and Deschênes, 2007). It is possible that these GJ-coupled clusters spread across multiple functional domains (e.g., domains for more than one whisker), but our interpretation is consistent with structure-function studies of TRN that show a low incidence of whiskerevoked activity spreading to neighboring TRN domains (Desîlets-Roy et al., 2002). The functional nature of all neurons in a single cluster could be answered by identifying all of their axonal projections, but this was not feasible because of the weak axonal staining of coupled neurons.

If coupled neurons of elongated or discoid clusters belong to a single functional domain of the TRN, the GJ coupling in clusters is likely to promote the spread of electrical and chemical signals and spike synchronization between subsets of TRN neurons that target a single domain in relay nuclei. Transient excitatory inputs to small parts of a coupled cluster could induce signal spread and recruitment of coupled neurons (Deans et al., 2002; Parker et al., 2009), leading to enhanced feedback inhibition or lateral inhibition within a limited domain of a relay nucleus. During periods of prolonged excitation over a whole TRN cluster, GJs would facilitate synchronized spiking in the TRN and thus enhance phasic inhibition and entrainment of spike times in relay neurons over the domain (Beierlein et al., 2000; Long et al., 2004). This entrainment could contribute to the synchrony of sensory-evoked spikes in thalamic relay neurons, a factor important in TC information transmission (Alonso et al., 1996; Bruno and Sakmann, 2006).

In contrast to the elongated or discoid clusters, spherical clusters of TRN neurons spread their neurons broadly across the short axis of the TRN. This pattern would synaptically connect neurons in different TRN tiers, either between neurons in adjacent domains for different body parts in the VB or between domains in the VB and POm. The injected neurons of spherical clusters tend to have branched axons with dual projection patterns to both $\mathrm{VB}$ and $\mathrm{POm}$, indicating they provide synchronized inhibition to the two nuclei. It is unclear whether coupled neurons in this cluster type are exclusively neurons with branched axons. This circuit seems an effective pathway for inhibitory control of the $\mathrm{VB}$ and POm by integrated 
activity of multiple tiers in the TRN. Although possible functions of this circuit are unknown, synaptic interactions via spherical clusters and divergent axonal projections could account for observations of reciprocal inhibitory interaction between the $\mathrm{VB}$ and POm (Crabtree et al., 1998) and of TRN neurons that receive convergent inputs from a wide thalamic area (Lam and Sherman, 2011).

It is not clear from our data whether the dye-coupled clusters we detected are part of larger GJ-coupled networks and whether different cluster patterns are connected via second-order coupling. Such putative undetected second-order couplings would be probably very weakly connected to the injected neurons. However, if there is global excitation over a wide area of the TRN, connected networks could play a significant role and could contribute to the generation of large-scale synchronous rhythmic activity in the thalamus (Beenhakker and Huguenard, 2009).

\section{Technical aspects of the dye-coupling method}

Technical improvements of the dye-injection method were critically important to this study. To deliver high concentrations of tracer into a target neuron without leakage to the extracellular space, we combined a front-filling technique with real-time monitoring of the fluorescence of the indicator LY. This approach should be broadly applicable for studying the spatial structure of GJ-coupled networks in many other brain areas.

One difficulty with our method is the precise control of dye concentration during intracellular injection compared with using a known and homogeneous concentration of dye in the pipette. With the front-filling strategy, dye diffused to the pipette tip during the waiting period. Although significant dye concentration could be reached reliably within a reasonable time, dye concentration in the tip did not reach that of the backfilling solution even after long waits. The LY signal was used to estimate when the intended NB concentration was reached. However, variability in $\mathrm{NB}$ concentrations inevitably arose from inaccuracies in fluorescence measurement, light scattering in the tissue, and cell morphology. More accurate measurement of LY fluorescence would improve the technique.

One alternative method of dye delivery is sharp microelectrodes. Sharp electrodes have long been used for dye injections into neurons (Gutnick and Prince, 1981; Connors et al., 1983; Hampson et al., 1992; Placantonakis et al., 2004), although they can generate false-positive signals attributable to dye leakage combined with cell membrane damage (Connors et al., 1984; Gutnick et al., 1985; Peinado et al., 1993). It would be interesting to test the feasibility of visually guided sharp electrode recordings, which minimize false positives in the retina (Pan et al., 2010). Laser-induced glutamate uncaging is another approach to mapping the spatial organization of GJ networks (Deleuze and Huguenard, 2006; Lam et al., 2006). Glutamate uncaging and dye coupling are complementary tools.

\section{Dye coupling versus electrical coupling}

The dye-coupling technique reveals intercellular GJ pathways that are permeable to NB molecules. Detecting coupled neurons depends on numerous factors, including the concentration of $\mathrm{NB}$ in the injected cells, the diffusion rate of NB through the cytoplasm, its permeability through the plasma membrane, and its permeability through GJ channels. If we assume that cytoplasmic diffusion of NB is fast and NB leakage through the plasma membrane is negligible, the dye-coupling signal is mainly determined by the rate of dye diffusion across GJ channels, which further depends on the number and subtypes of GJ channels (Mills and
Massey, 1998). Although heterogeneous GJ channel subtypes or permeabilities could complicate this relationship (Eckert, 2006), it seems very likely that the intensity of dye coupling is positively correlated with the junctional conductance (Verselis et al., 1986).

Paired-cell recordings suggested that the prevalence of coupling is high between closely spaced TRN neurons but drops rapidly to zero beyond intersomatic distances of $\sim 60 \mu \mathrm{m}$ (Long et al., 2004). Our dye coupling showed a much wider range of intersomatic distances $(10-340 \mu \mathrm{m})$. This discrepancy probably reflects the different natures of the techniques. Paired recording is a powerful method for detecting closely spaced coupled pairs but inefficient for finding rare GJ-coupled neurons interspersed with many uncoupled cells across long distances, especially with cells distributed anisotropically. However, paired recordings seem to be more sensitive indicators of coupling; the prevalence of electrical coupling is $\sim 50 \%$ at short intersomatic distances $(<20 \mu \mathrm{m})$, whereas the density of dye-coupled neurons is lower at these distances. This may explain apparent discrepancies in the data acquired from WT and Cx36 KO mice using the two techniques.

\section{Molecular heterogeneity of TRN GJ coupling}

Dye coupling in Cx36-deficient TRN was substantially reduced compared with WT, suggesting that most NB dye coupling occurred through Cx36-containing GJs. This is consistent with the $\mathrm{Cx} 36$ dependence of NB dye coupling in neurons of the retina and inferior olive (Placantonakis et al., 2004; Pan et al., 2010). Our observations of significant residual dye coupling and electrical coupling between TRN neurons of $\mathrm{Cx} 36 \mathrm{KO}$ mice are surprising. Previous studies of $\mathrm{Cx} 36 \mathrm{KO}$ mice reported the absence, or near absence, of electrical coupling in a variety of neuron types that are normally coupled at high rates, including interneurons of the neocortex, neurons in the retina, the olfactory bulb, and the inferior olivary nucleus (Deans et al., 2001, 2002; Long et al., 2002; Christie et al., 2005). The steep distance dependence of coupling as measured by paired recording and differences of experimental conditions (e.g., intersomatic distance, recording area, and age) may account for the very low incidence of electrical coupling in a previous study of the TRN in Cx36 KO mice from our laboratory (Landisman et al., 2002). In our current study, residual coupling in the $\mathrm{KO}$ clearly indicates the presence of functional GJ proteins other than Cx36, as reported in some other studies (Pan et al., 2010; Lee et al., 2010; Curti et al., 2012). It will be important to elucidate the molecular basis of non-Cx36 GJs. Although Cx36-independent coupling in the TRN is sparse and weak, its existence suggests that experiments using $\mathrm{Cx} 36 \mathrm{KO}$ mice should be carefully interpreted.

\section{References}

Agmon A, Connors BW (1991) Thalamocortical responses of mouse somatosensory (barrel) cortex in vitro. Neuroscience 41:365-379. CrossRef Medline

Alonso JM, Usrey WM, Reid RC (1996) Precisely correlated firing in cells of the lateral geniculate nucleus. Nature 383:815-819. CrossRef Medline

Amitai Y, Gibson JR, Beierlein M, Patrick SL, Ho AM, Connors BW, Golomb D (2002) The spatial dimensions of electrically coupled networks of interneurons in the neocortex. J Neurosci 22:4142-4152. Medline

Beenhakker MP, Huguenard JR (2009) Neurons that fire together also conspire together: is normal sleep circuitry hijacked to generate epilepsy? Neuron 62:612-632. CrossRef Medline

Beierlein M, Gibson JR, Connors BW (2000) A network of electrically coupled interneurons drives synchronized inhibition in neocortex. Nat Neurosci 3:904-910. CrossRef Medline

Benardo LS (1997) Recruitment of GABAergic inhibition and synchroniza- 
tion of inhibitory interneurons in rat neocortex. J Neurophysiol 77:31343144. Medline

Bourassa J, Pinault D, Deschênes M (1995) Corticothalamic projections from the cortical barrel field to the somatosensory thalamus in rats: a single-fibre study using biocytin as an anterograde tracer. Eur J Neurosci 7:19-30. CrossRef Medline

Bruno RM, Sakmann B (2006) Cortex is driven by weak but synchronously active thalamocortical synapses. Science 312:1622-1627. CrossRef Medline

Buhl DL, Harris KD, Hormuzdi SG, Monyer H, Buzsáki G (2003) Selective impairment of hippocampal gamma oscillations in connexin-36 knockout mouse in vivo. J Neurosci 23:1013-1018. Medline

Bukauskas FF (2012) Neurons and $\beta$-cells of the pancreas express connexin36, forming gap junction channels that exhibit strong cationic selectivity. J Membr Biol 245:243-253. CrossRef Medline

Christie JM, Bark C, Hormuzdi SG, Helbig I, Monyer H, Westbrook GL (2005) Connexin 36 mediates spike synchrony in olfactory bulb glomeruli. Neuron 46:761-772. CrossRef Medline

Connors BW, Long MA (2004) Electrical synapses in the mammalian brain. Annu Rev Neurosci 27:393-418. CrossRef Medline

Connors BW, Benardo LS, Prince DA (1983) Coupling between neurons of the developing rat neocortex. J Neurosci 3:773-782. Medline

Connors BW, Benardo LS, Prince DA (1984) Carbon dioxide sensitivity of dye coupling among glia and neurons of the neocortex. J Neurosci 4:1324-1330. Medline

Crabtree JW (1992) The somatotopic organization within the rabbit's thalamic reticular nucleus. Eur J Neurosci 4:1343-1351. CrossRef Medline

Crabtree JW, Collingridge GL, Isaac JT (1998) A new intrathalamic pathway linking modality-related nuclei in the dorsal thalamus. Nat Neurosci 1:389-394. CrossRef Medline

Cruikshank SJ, Hopperstad M, Younger M, Connors BW, Spray DC, Srinivas M (2004) Potent block of Cx36 and Cx50 gap junction channels by mefloquine. Proc Natl Acad Sci U S A 101:12364-12369. CrossRef Medline

Curti S, Hoge G, Nagy JI, Pereda AE (2012) Synergy between electrical coupling and membrane properties promotes strong synchronization of neurons of the mesencephalic trigeminal nucleus. J Neurosci 32:4341-4359. CrossRef Medline

Deans MR, Gibson JR, Sellitto C, Connors BW, Paul DL (2001) Synchronous activity of inhibitory networks in neocortex requires electrical synapses containing connexin36. Neuron 31:477-485. CrossRef Medline

Deans MR, Volgyi B, Goodenough DA, Bloomfield SA, Paul DL (2002) Connexin 36 is essential for transmission of rod-mediated visual signals in the mammalian retina. Neuron 36:703-712. CrossRef Medline

Deleuze C, Huguenard JR (2006) Distinct electrical and chemical connectivity maps in the thalamic reticular nucleus: potential roles in synchronization and sensation. J Neurosci 26:8633-8645. CrossRef Medline

Deschênes M, Veinante P, Zhang ZW (1998) The organization of corticothalamic projections: reciprocity versus parity. Brain Res Brain Res Rev 28:286-308. CrossRef Medline

Desîlets-Roy B, Varga C, Lavallée P, Deschênes M (2002) Substrate for cross-talk inhibition between thalamic barreloids. J Neurosci 22: RC218(1-4). Medline

Dugué GP, Brunel N, Hakim V, Schwartz E, Chat M, Lévesque M, Courtemanche R, Léna C, Dieudonné S (2009) Electrical coupling mediates tunable low-frequency oscillations and resonance in the cerebellar Golgi cell network. Neuron 61:126-139. CrossRef Medline

Eckert R (2006) Gap-junctional single-channel permeability for fluorescent tracers in mammalian cell cultures. Biophys J 91:565-579. CrossRef Medline

Galarreta M, Hestrin S (2001a) Electrical synapses between GABA-releasing interneurons. Nat Rev Neurosci 2:425-433. CrossRef Medline

Galarreta M, Hestrin S (2001b) Spike transmission and synchrony detection in networks of GABAergic interneurons. Science 292:2295-2299. CrossRef Medline

Gibson JR, Beierlein M, Connors BW (1999) Two networks of electrically coupled inhibitory neurons in neocortex. Nature 402:75-79. CrossRef Medline

Gutnick MJ, Prince DA (1981) Dye coupling and possible electrotonic coupling in the guinea pig neocortical slice. Science 211:67-70. CrossRef Medline

Gutnick MJ, Lobel-Yaakov R, Rimon G (1985) Incidence of neuronal dye- coupling in neocortical slices depends on the plane of section. Neuroscience 15:659-666. CrossRef Medline

Hampson EC, Vaney DI, Weiler R (1992) Dopaminergic modulation of gap junction permeability between amacrine cells in mammalian retina. J Neurosci 12:4911-4922. Medline

Hjorth J, Blackwell KT, Kotaleski JH (2009) Gap junctions between striatal fast-spiking interneurons regulate spiking activity and synchronization as a function of cortical activity. J Neurosci 29:5276-5286. CrossRef Medline

Kamholz AE, Schilling EA, Yager P (2001) Optical measurement of transverse molecular diffusion in a microchannel. Biophys J 80:1967-1972. CrossRef Medline

Kimura A, Donishi T, Okamoto K, Tamai Y (2005) Topography of projections from the primary and non-primary auditory cortical areas to the medial geniculate body and thalamic reticular nucleus in the rat. Neuroscience 135:1325-1342. CrossRef Medline

Lam YW, Sherman SM (2007) Different topography of the reticulothalmic inputs to first- and higher-order somatosensory thalamic relays revealed using photostimulation. J Neurophysiol 98:2903-2909. CrossRef Medline

Lam YW, Sherman SM (2011) Functional organization of the thalamic input to the thalamic reticular nucleus. J Neurosci 31:6791-6799. CrossRef Medline

Lam YW, Nelson CS, Sherman SM (2006) Mapping of the functional interconnections between thalamic reticular neurons using photostimulation. J Neurophysiol 96:2593-2600. CrossRef Medline

Landisman CE, Long MA, Beierlein M, Deans MR, Paul DL, Connors BW (2002) Electrical synapses in the thalamic reticular nucleus. J Neurosci 22:1002-1009. Medline

Lee SC, Cruikshank SJ, Connors BW (2010) Electrical and chemical synapses between relay neurons in developing thalamus. J Physiol 588:24032415. CrossRef Medline

Lin JY, van Wyk M, Bowala TK, Teo MY, Lipski J (2003) Dendritic projections and dye-coupling in dopaminergic neurons of the substantia nigra examined in horizontal brain slices from young rats. J Neurophysiol 90: 2531-2535. CrossRef Medline

Long MA, Deans MR, Paul DL, Connors BW (2002) Rhythmicity without synchrony in the electrically uncoupled inferior olive. J Neurosci 22: 10898-10905. Medline

Long MA, Landisman CE, Connors BW (2004) Small clusters of electrically coupled neurons generate synchronous rhythms in the thalamic reticular nucleus. J Neurosci 24:341-349. CrossRef Medline

Mills SL, Massey SC (1998) The kinetics of tracer movement through homologous gap junctions in the rabbit retina. Vis Neurosci 15:765-777. Medline

Mills SL, Massey SC (2000) A series of biotinylated tracers distinguishes three types of gap junction in retina. J Neurosci 20:8629-8636. Medline

Pan F, Paul DL, Bloomfield SA, Völgyi B (2010) Connexin36 is required for gap junctional coupling of most ganglion cell subtypes in the mouse retina. J Comp Neurol 518:911-927. CrossRef Medline

Parker PR, Cruikshank SJ, Connors BW (2009) Stability of electrical coupling despite massive developmental changes of intrinsic neuronal physiology. J Neurosci 29:9761-9770. CrossRef Medline

Peinado A, Yuste R, Katz LC (1993) Extensive dye coupling between rat neocortical neurons during the period of circuit formation. Neuron 10: 103-114. CrossRef Medline

Petrásek Z, Schwille P (2008) Precise measurement of diffusion coefficients using scanning fluorescence correlation spectroscopy. Biophys J 94:14371448. CrossRef Medline

Pierret T, Lavallée P, Deschênes M (2000) Parallel streams for the relay of vibrissal information through thalamic barreloids. J Neurosci 20:74557462. Medline

Pinault D (2004) The thalamic reticular nucleus: structure, function and concept. Brain Res Brain Res Rev 46:1-31. CrossRef Medline

Pinault D, Bourassa J, Deschênes M (1995) The axonal arborization of single thalamic reticular neurons in the somatosensory thalamus of the rat. Eur J Neurosci 7:31-40. CrossRef Medline

Placantonakis DG, Bukovsky AA, Zeng XH, Kiem HP, Welsh JP (2004) Fundamental role of inferior olive connexin 36 in muscle coherence during tremor. Proc Natl Acad Sci U S A 101:7164-7169. CrossRef Medline

Rozental R, Srinivas M, Spray DC (2001) How to close a gap junction channel: efficacies and potencies of uncoupling agents. In: Methods in molec- 
ular biology, connexin methods and protocols (Bruzzone R, Giaume C, eds), pp 447-476. Totowa, NJ: Humana.

Schlösser B, ten Bruggencate G, Sutor B (1998) The intracellular tracer Neurobiotin alters electrophysiological properties of rat neostriatal neurons. Neurosci Lett 249:13-16. CrossRef Medline

Shosaku A, Kayama Y, Sumitomo I (1984) Somatotopic organization in the rat thalamic reticular nucleus. Brain Res 311:57-63. CrossRef Medline

Spector R, Johanson CE (2007) Vitamin transport and homeostasis in mammalian brain: focus on Vitamins B and E. J Neurochem 103:425438. CrossRef Medline

Stewart WW (1978) Functional connections between cells as revealed by dye-coupling with a highly fluorescent naphthalimide tracer. Cell 14:741759. CrossRef Medline

Tiesinga P, Fellous JM, Sejnowski TJ (2008) Regulation of spike timing in visual cortical circuits. Nat Rev Neurosci 9:97-107. CrossRef Medline

Traub RD, Kopell N, Bibbig A, Buhl EH, LeBeau FE, Whittington MA (2001)

Gap junctions between interneuron dendrites can enhance synchrony of gamma oscillations in distributed networks. J Neurosci 21:9478-9486. Medline

Urbain N, Deschênes M (2007) A new thalamic pathway of vibrissal information modulated by the motor cortex. J Neurosci 27:12407-12412. CrossRef Medline

Verselis V, White RL, Spray DC, Bennett MV (1986) Gap junctional conductance and permeability are linearly related. Science 234:461-464. CrossRef Medline

Vervaeke K, Lorincz A, Gleeson P, Farinella M, Nusser Z, Silver RA (2010) Rapid desynchronization of an electrically coupled interneuron network with sparse excitatory synaptic input. Neuron 67:435-451. CrossRef Medline

Wimmer VC, Bruno RM, de Kock CP, Kuner T, Sakmann B (2010) Dimensions of a projection column and architecture of VPM and POm axons in rat vibrissal cortex. Cereb Cortex 20:2265-2276. CrossRef Medline

Xi XZ, Xu ZC (1996) The effect of neurobiotin on membrane properties and morphology of intracellularly labeled neurons. J Neurosci Methods 65: 27-32. CrossRef Medline 